\title{
DESIGN CRITERIA FOR MULTILAYER SUPERCONDUCTIYE MAGNETS
}

M. N. El Derini, ${ }^{*+}$ M. A. Hilal* and R. W. Boom*

\section{ABSTRACT}

High current round composite conductors with superconductors near the surface are under development at the University of Wisconsin. The conductors are designed for single layer energy storage solenoids and possibly for solenoids with several layers. The radial and axial forces in such magnets are obtained by summing forces between turns. Solenoids with constant tension are achieved by changing the spacing between conductors in the axial direction. Multilayer solenoids are designed so that the tension in the different layers is the same as the required design value. This design value is chosen to make the conductors remain in tension. Constant tension designs facilitate the economic use of force-bearing structure in energy storage and fusion superconductive magnets.

\section{INTRODUCTION}

Large thin superconducting solenoids are needed for energy storage applications and for poloidal coils in Tokamak reactors. For energy storage, single or multilayer solenoids are needed with forces transmitted to bedrock by means of insulating struts. It is the purpose of this study to design multilayer solenoids using the Wisconsin round conductor and to compare this design to the previous single layer design.? The conductors must remain in tension to prevent any compressive structural instability which may occur in the outer layers of the magnet. It is, therefore, necessary for al 1 radial components of the magnetic forces to point away from the axis of the solenoid. Multilayer solenoids are designed so that the tension in the different layers is the same as the required design value to make full use of the conductor.

\section{PROCEDURE}

A virtual displacement technique is used to calculate the forces on the turns. It has the advantage of avoiding the mathematical difficulties in calculating fields (and hence forces) inside the windings.

Forces in various directions are equal to energy derivatives in those directions. We generalize by calculating mutual inductances between superconductor filaments in different turns. Forces are found for a round energy storage conductor which is $8 \mathrm{~cm}$ in dia. with a layer of $6 \times 10^{5}$ superconductor filaments on a $7.5 \mathrm{~cm}$ dia.

The mutual inductance between two round conductors, Fig. 1 , is given by

Manuscript received September 28, 1978.

* University of Wisconsin, 1500 Johnson Drive, Madison, Wisconsin

+ This work was initiated as part of a Ph.D. dissertation in the Electrical Engineering Department, University of Wisconsin, Madison.

$$
\begin{aligned}
& M=10^{-4} \int_{0}^{2 \pi} \int_{0}^{2 \pi} f\left(k^{\prime 2}\right) \sqrt{r_{1} r_{2}} d \theta_{1} d \theta_{2} \text { henries. } \\
& f\left(k^{\prime 2}\right) \text { is assumed to have the following form: } \\
& f\left(k^{\prime 2}\right)=\sum_{n} C_{n}\left(\ln \left(k^{\prime 2}\right)\right)^{n},
\end{aligned}
$$

where $k^{\prime 2}$ is

$$
\begin{aligned}
& k^{\prime 2}=\left\{\left(1-\alpha_{1}\right)^{2}+\alpha_{2}{ }^{2}+\alpha_{3}{ }^{3}+\alpha_{4}{ }^{2}+2 \alpha_{4}\left(1-\alpha_{1}\right) \cos \theta_{2}\right. \\
&+2 \alpha_{2} \alpha_{4} \sin \theta_{2}-2 \alpha_{3}\left(1-\alpha_{1}\right) \cos \theta_{1}-2 \alpha_{2} \alpha_{3} \sin \theta_{1} \\
&\left.-2 \alpha_{3} \alpha_{4} \cos \left(\theta_{1}-\theta_{2}\right)\right\} /\left\{\left(1+\alpha_{1}\right)^{2}+\alpha_{2}{ }^{2}\right\}, \\
& \alpha_{1}=r_{1} / r_{2}, a_{2}=d / r_{2}, a_{3}=a_{1} / r_{2}, \text { and } \alpha_{4}=a_{2} / r_{2},
\end{aligned}
$$

using the tabulated values for $f$ in reference 3 , the coefficients $C_{n}$ are calculated.

The energy stored by two round conductors is

$$
\begin{gathered}
E=J_{1} J_{2} \iint M_{12} d \ell_{1} d l_{2}+\frac{1}{2} J_{1}^{2} \iint L_{1} d \ell_{1} d \ell_{1}{ }^{\prime} \\
+\frac{1}{2} J_{2}^{2} \iint L_{2} d \ell_{2} d l_{2}{ }^{\prime}
\end{gathered}
$$

where $\mathrm{J}_{1}$ and $\mathrm{J}_{2}$ are the current per filament of length $\mathrm{d}_{1}$ or $\mathrm{d}_{2}$.

The radial and axial forces between two round conductors are calculated as the summation of forces between filaments:

$$
F_{r}\left(r_{1}, z_{1}\right)=\frac{I_{1} I_{2} \times 10^{-4}}{8 \pi^{3} r_{1}} \int_{0}^{2 \pi} \int_{0}^{2 \pi} \frac{d M_{12}}{d r_{1}} d \theta_{1} d \theta_{2},
$$

and

$$
F_{z}\left(r_{1}, z_{1}\right)=\frac{I_{1} I_{2} \times 10^{-4}}{8 \pi^{3} r_{1}} \int_{0}^{2 \pi} \int_{0}^{2 \pi} \frac{d M_{12}}{d z_{1}} d \theta_{1} d \theta_{2},
$$

where $I_{1}$ and $I_{2}$ are the currents carried by the first and the second conductor, and

$$
\begin{aligned}
& \frac{d M_{12}}{d r_{1}}=.5 \sqrt{\frac{r_{2}}{r_{1}}} f\left(k^{\prime 2}\right)+\sqrt{r_{1} r_{2}}, \frac{\partial f\left(k^{\prime 2}\right)}{\partial k^{\prime 2}}, \\
& \frac{d M_{12}}{d z_{1}}=\sqrt{r_{1} r_{2}} \zeta_{2} \frac{\partial f\left(k^{\prime 2}\right)}{\partial k^{\prime 2}},
\end{aligned}
$$

where 


$$
\begin{aligned}
\zeta_{1}= & -2\left(2\left(1-\alpha_{1}{ }^{2}+\alpha_{2}{ }^{2}\right)+\left(1+\alpha_{1}\right)\left(\alpha_{3}{ }^{2}+\alpha_{4}{ }^{2}\right)\right. \\
& +\left(3+2 \alpha_{1}-\alpha_{1}{ }^{2}+\alpha_{2}{ }^{2}\right)\left(\alpha_{4} \cos \theta_{2}-\alpha_{3} \cos \theta_{1}\right) \\
& \left.+2\left(1+\alpha_{1}\right)\left[\alpha_{1} \alpha_{4} \sin \theta_{2}-\alpha_{2} \alpha_{3} \sin \theta_{1}-\alpha_{3} \alpha_{4} \cos \left(\theta_{1}-\theta_{2}\right)\right]\right\} / \\
& r_{2}\left[\left(1+\alpha_{1}\right)^{2}+\alpha_{2}{ }^{2}\right]^{2}
\end{aligned}
$$

and

$$
\begin{aligned}
\zeta_{2}= & 2\left\{\alpha_{2}\left(4 \alpha_{1}-\alpha_{3}{ }^{2}-\alpha_{4}{ }^{2}\right)+\left(\left(1+\alpha_{1}\right)^{2}-\alpha_{2}{ }^{2}\right)\left(\alpha_{4} \sin \theta_{2}-\alpha_{3} \sin \theta_{1}\right)\right. \\
& \left.-2 \alpha_{2}\left[\left(1-\alpha_{1}\right)\left(\alpha_{4} \cos \theta_{2}-\alpha_{3} \cos \theta_{1}\right)-\alpha_{3} \alpha_{4} \cos \left(\theta_{1}-\theta_{2}\right)\right]\right\} / \\
& r_{2}\left[\left(1+\alpha_{1}\right)^{2}+\alpha_{2}{ }^{2}\right]^{2} .
\end{aligned}
$$

The force on each conductor is the sum of the forces due to all the other conductors. A comparison between the forces calculated by (6) and (7) with the forces due to the replacement of a round conductor by a filament at the center is done as a function of the axial distance between conductors for different radi $i$ of the solenoid. The maximum percentage error is less than one percent. The forces are calculated by replacing each round conductor by a filament at its center.

Forces are optimized to obtain constant tension in a1 1 conductors in the same layer, by varying the current distribution axially in each layer. The current distribution per unit length in each layer, $J_{j}(z)$, is represented by Legendre polynomials

$$
\begin{aligned}
u_{i}(z)= & A_{i}\left(a_{0}+0.5\left(3(z / \ell)^{2}-1\right) a_{2}\right. \\
& \left.+0.125\left(35(z / \ell)^{4}-30(z / \ell)^{2}+3\right) a_{4}\right),
\end{aligned}
$$

where $A_{j}$ is the fraction of the current in the ith layer, $a_{0}, a_{2}$ and $a_{4}$ are Legendre coefficients. The same coefficients $a_{0}, a_{2}$ and $a_{4}$ are assumed, which results in a practical current distribution where the position of turns in the layers is the same.

The Legendre coefficients $a_{0}, a_{2}$ and $a_{4}$ are obtained using the Teast-squares method. The current is divided between two or three layers. The radial force per unit ampere is a function of the fractions of the current in each layer $A_{j}$. To have a constant radial force per unit ampere, for all conductors in the ith layer requires

$$
\text { minimizing } \sum_{z}\left\{\sum_{i}\left(F_{r_{i}}\left(z, A_{i}\right)-B_{i}\right)^{2}\right\} \text {, }
$$

where $B_{i}$ is the required tension in the $i$ th layer, and

$$
\sum_{i}^{N} B_{i}=1 \text {. }
$$

\section{OPTIMIZATION CONSIDERING ALL VARIABLES}

We solve Eq. (13) considering $A_{1}, A_{2}, \ldots, A_{N}, B_{1}$, $B_{2}, \ldots, B_{N-1}$ as independent variables. We search for the opt imal point $\left(\hat{A}_{1}, \hat{A}_{2}, \ldots, \hat{A}_{N}, \hat{B}_{1}, \ldots, \hat{B}_{N-1}\right)$ using the Davidon Fletcher-Powe11 (DFP) method. We consider a two layer solenoid which is optimized in three variables, and a three layer solenoid which is optimized in five variables. Two examples are considered; the University of Wisconsin energy storage solenoid and the KEK, the National Laboratory for High Energy Physics, Japan's solenoid. The current and tension distributions are tabulated (Table 1) for the examples for one, two and three layer magnets. The optimal solution is found to be impractical because the current changes direction from layer to layer,

\begin{tabular}{|c|c|c|c|c|c|c|c|}
\hline \multirow{2}{*}{\multicolumn{2}{|c|}{$\begin{array}{l}\text { No. of } \\
\text { Layer }\end{array}$}} & \multicolumn{3}{|c|}{ Current Distribution } & \multirow{2}{*}{$\begin{array}{c}\text { Tension } \\
15 t\end{array}$} & \multicolumn{2}{|c|}{ Distribution } \\
\hline & & ist & 2nd & 3rd & & $2 n d$ & $3 r d$ \\
\hline \multirow{3}{*}{ 㕸 } & 1 & 1 & - & - & $T$ & - & - \\
\hline & 2 & 0.0064 & 0.9936 & - & 0.025 & 0.975 & - \\
\hline & 3 & 0.0221 & 0.0025 & 0.9754 & 0.078 & 0.009 & 0.913 \\
\hline \multirow{3}{*}{$\tilde{\omega}$} & 1 & 1 & 1 & - & - & 1 & - \\
\hline & 2 & 0.0005 & 0.9995 & - & 0.003 & 0.997 & - \\
\hline & 3 & 0.0004 & 0.9998 & -.0002 & 0.002 & 0.997 & 0.001 \\
\hline
\end{tabular}
which results in an inefficient energy storage system. Furthermore, conductors in the outer layer of the magnet will be in compression, which is

undesirable. It is important to notice that the major fraction of the current will be carried in one layer. To achieve more uniform tension in all turns, one may consider other layers as only correction coils.

Table 1

Optimal Current and Optima? Tension Distributions for One, Two and Three Layer Solenoids

The currents should also be in the same direction in all layers even if all the conductors are in tension. To achieve this design, we vary the tension coefficients $B_{i} ' s$, and for different combinations of $B_{i}$ 's, we minimize Eq. (13) to obtain the optimum values of $A_{j}{ }^{\prime} s$. The normalized radial forces as a function of a dimensionless axial distance are shown in Figs. 2-8. Normalized current per unit length and normalized radial force for a one layer magnet are shown in Fig. 2. More currents and hence more turns are needed at the end of the solenoid than at the midplane to achieve a constant tension solenoid. Normalized total radial forces for two and three layer KEK and ES solenoids are shown in Figs. 3, 4. These forces are for constant tension and uniform current solenoids. The radial force is not exactly constant for the constant tension solenoid but is less variable than for a uniform current solenoid. The normalized radial force on the first, second, and third layers are shown in Figs. 5 and 7 for both constant tension and uniform current solenoids. The normalized radial forces on each layer are shown in Figs. 6, 8 for uniform current solenoids. The forces are normalized to the average force, $F_{a v}$, on each layer. The current and tension distributions taken for the previous seven figures are tabulated in Table 2. Tension distribution is assumed so that a conductor remains in tension. The current does not change direction from one layer to another and is not concentrated in one layer only. The choice of the tension distribution must be based on cost optimization as well as on practicalities. Since the current distribution varies from layer to layer, conductors of different sizes are used in a multilayer magnet. An approximate calculation is used to determine the conductor sizes. 
316

Disregarding the area of the superconductors, we have

$$
A_{H S}+A_{H P}=\pi R^{2}
$$

where $A_{H S}$ and $A_{H P}$ are the areas of high strength and the high purity metals.

By solving Eq. (15)

$R_{i}=2 \sqrt{\frac{I_{i}^{2} R_{c u r}}{3 f_{i} \sigma \pi}} \cos \left(\cos ^{-1}\left(\frac{\rho}{4 \pi^{2} q I_{i}} \sqrt{\left.\left.\frac{27 f_{j}^{3 \sigma^{3} \pi^{3}}}{R_{c u r}^{3}}\right) / 3\right)}\right.\right.$

where $I_{i}$ is the current in a conductor of radius $R_{i}$ at layer $i ; f_{j}$ is the tension on this conductor; $R_{c m}$ is the average radius of curvature; $\sigma$ is the average stress in the high strength aluminum; $\rho$ is the resis tivity of the high purity aluminum and $q$ is the allowable surface heat flux.

An example for a two and three layer energy. storage magnet is

$$
\begin{array}{rlrl}
I_{\text {total }} & =157,000 \mathrm{~A}, \quad \rho=10^{-10} \Omega \mathrm{m} \\
\mathrm{R}_{\mathrm{Cm}} & =1.5 \mathrm{~m}, \quad \sigma=15,000 \mathrm{psi} \\
\mathrm{q} & =5000 \mathrm{w} / \mathrm{m}^{2}, &
\end{array}
$$

thus

$$
\begin{aligned}
& \mathrm{R}_{1}=1.222 \mathrm{~cm} \text { and } \mathrm{R}_{2}=1.370 \mathrm{~cm} \\
& \mathrm{R}_{1}=1.356 \mathrm{~cm}, \quad \mathrm{R}_{2}=1.031 \mathrm{~cm} \text { and } \mathrm{R}_{3}=1.348 \mathrm{~cm} .
\end{aligned}
$$

Table 2

Optimal Current and Assumed Tension Distributions for One, Two and Three Layer Solenoids

\begin{tabular}{cccc|ccc}
$\begin{array}{l}\text { No. of } \\
\text { Layers }\end{array}$ & \multicolumn{2}{c|}{ Current } & Distribution* & \multicolumn{4}{|c}{ Tersian } & Distribution** \\
\hline 1 & 1.0 & - & - & 1.0 & - & - \\
& 2nd & 3rd & 1st & 2nd & 3rd \\
\hline 2 & 0.175 & 0.825 & - & 0.6 & 0.4 & - \\
3 & 0.196 & 0.074 & 0.730 & 0.6 & 0.2 & 0.2 \\
& & & & & & \\
1 & 1.0 & - & - & 1.0 & - & - \\
2 & 0.1117 & 0.8883 & & 0.5 & 0.5 & - \\
3 & 0.127 & 0.062 & 0.811 & 0.5 & 0.25 & 0.25 \\
\hline
\end{tabular}

*alculated optimal current distribution

** Assumed tension distribution

\section{CONCLUSIONS}

The calculations of the mutual inductance between two round conductors, the forces and the energy stored in the magnets are discussed. In the calculations round conductors are replaced by filaments appropriately distributed. Constant tension multilayer solenoids are discussed as an application of the method. Diurnal and pulsed energy storage magnets are taken as examples.

The optimal design of a constant tension multilayer solenoid is not practical because most of the current is concentrated in one layer and sometimes the current changes its direction which decreases the energy stored in the magnet. On the other hand the optimal design leads to the use of correction coils, which is to add small coils to a constant tension one layer solenoid.

Some practical designs are presented in the paper. These designs are the optimal current distribution for a certain tension distribution which allows the conductor to be in tension without compressive structural instability conductors of slightly different sizes are used in a multilayer magnet using the energy storage magnet as an example.

\section{ACKNOWLEDGEMENT}

The KEK energy storage solenoid, which is used throughout the paper as an example, has resulted from KEK studies by M. Masuda, T. Shintomi, and S. Matsumoto. This work was supported by the U.S. Department of Energy.

REFERENCES

1. Wiscons in Superconductive Energy Storage Project, 1976 Annual Report, May 1, 1977.

2. M.N. El-Derini, R.H. Boom, M.A. Hilal, "Constent Tension and Constant Fieid Solenoids," to be published in Advances in Cryogenic Engineering, vol. 23 ,
1978, po. 89-96. 3. Grover, F. W., "Inductance Calculations, Working Fomulas and Tables," Dover
Publications, Inc., Wew rork, N.Y. (1962),

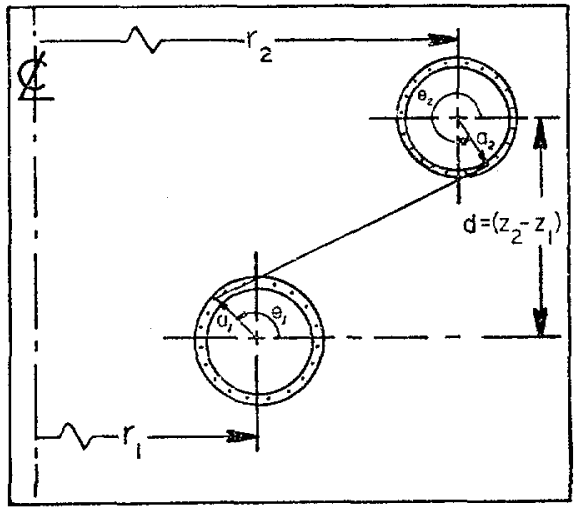

Fig. 1. Two round conductors with composite superconductors near the surface.

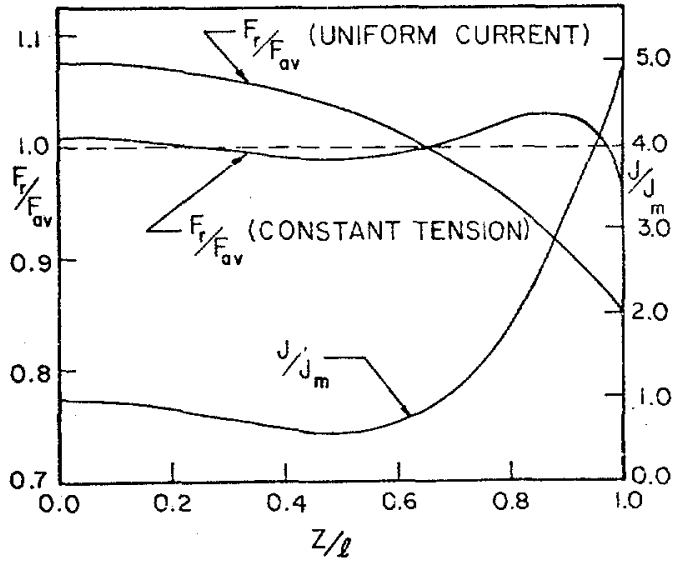

Fig. 2. Normalized radial force and normalized current per unit length for constant tension uniform current one layer solenoid. 


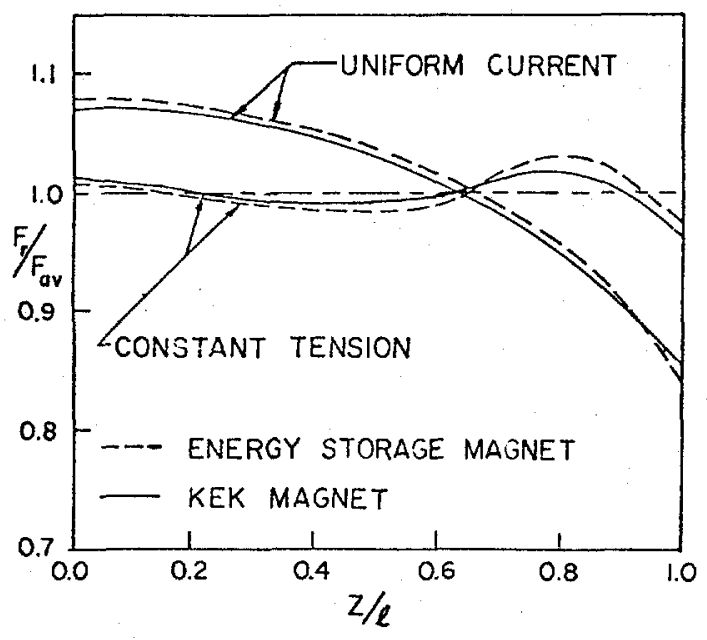

Fig. 3. Normalized total radial force for uniform current and constant tension two layer solenoids.

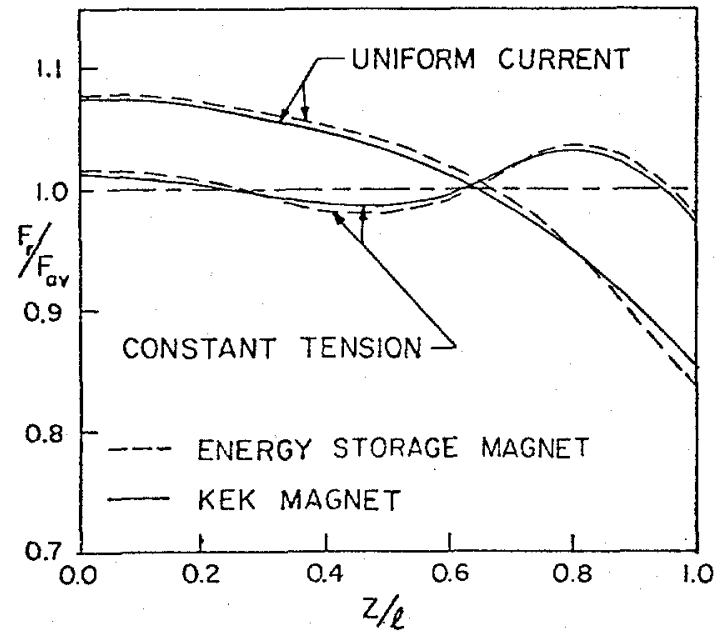

Fic. 4. Normalized total radial force for uniform current and constant tension three layer solenoids.

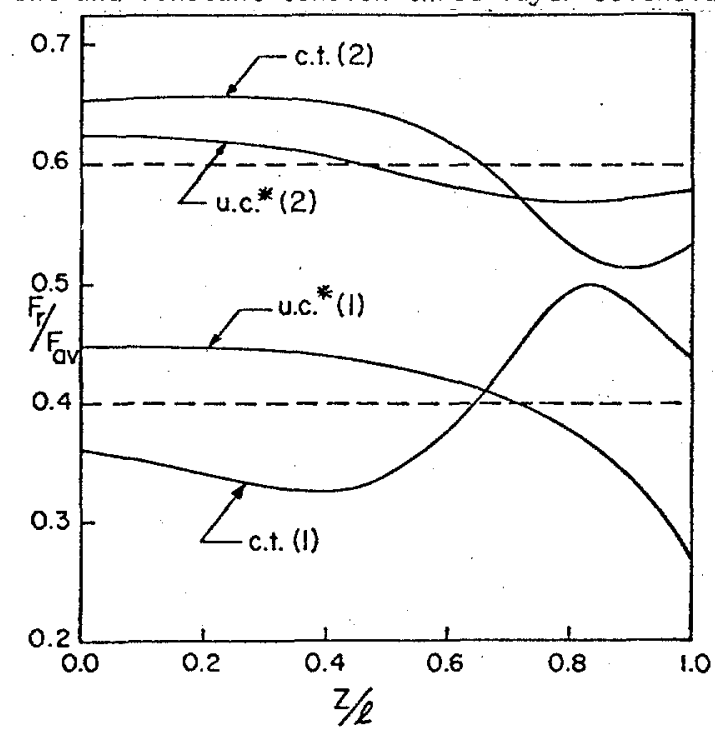

Fig. 5. Normalized radial force for constant tension and uniform current* two layer solenoids

c.t.(i) - constant tension in the ith layer

u.c.*(i) - uniform current with radial distribution of current as in constant tension, in the ith Tayer.

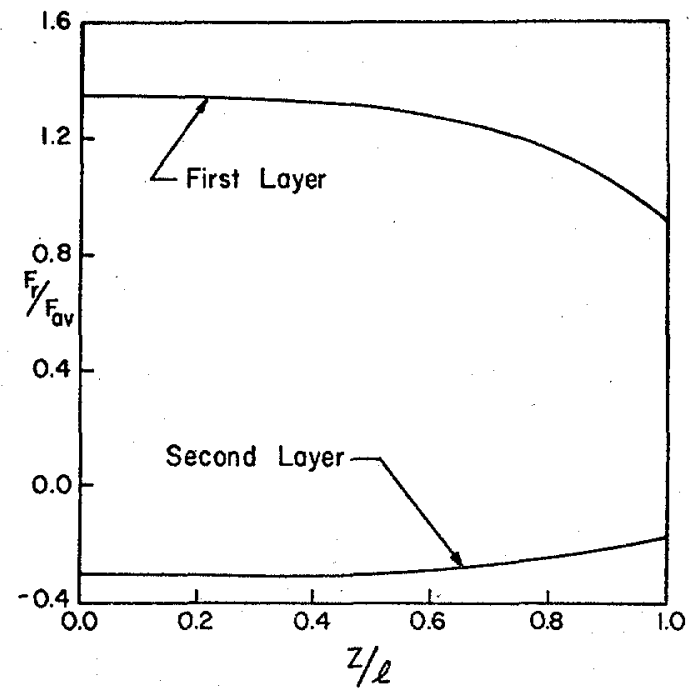

Fig. 6. Normalized radial force on each layer of uniform current two layer solenoid.

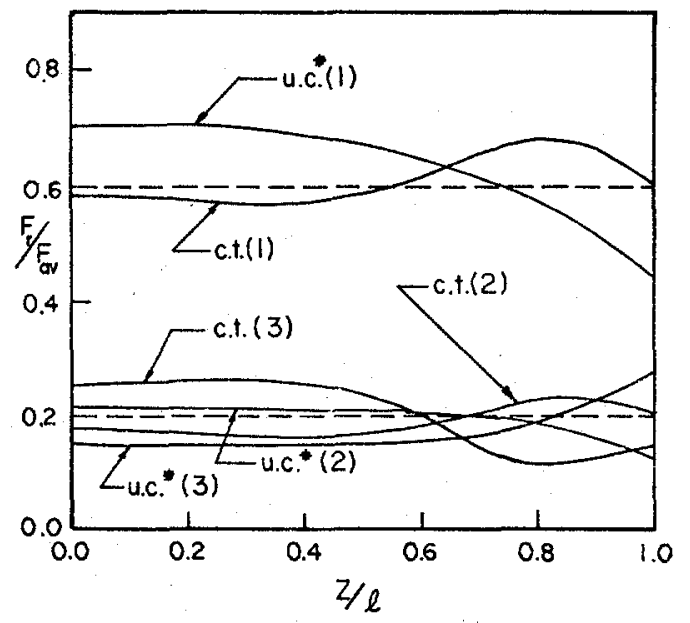

Fig. 7. Normalized radial force for constant tension and uniform current* three layer solenoids

c.t. (i) - constant tension in the ith layer

u.c.*(i) - uniform current, with radial distribution of current as in constant tension, in the ith layer

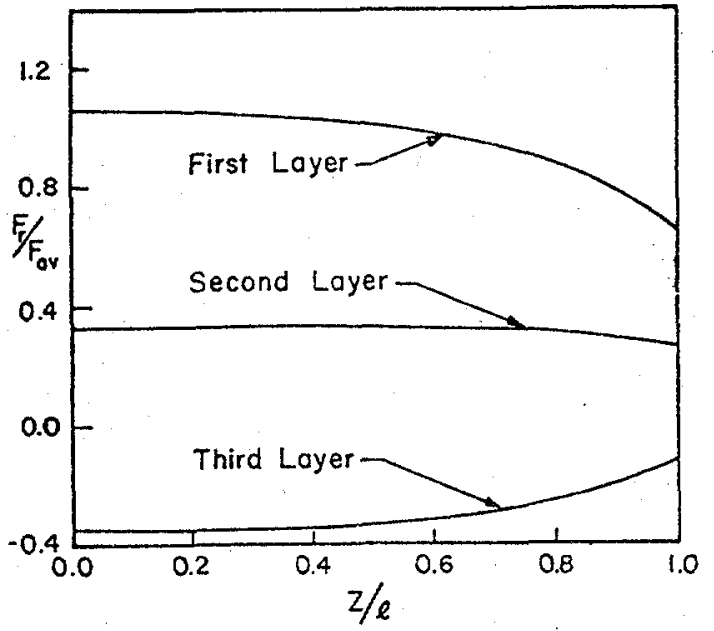

Fig. 8. Normalized radial force on each layer of uniform current three layer solenoid. 